\title{
Transtorno de escoriação: diagnóstico e intervenção - uma revisão sistemática
}

\author{
Hugo Tanizaka* \\ Amanda Giusti** \\ Karen Santos** \\ Rosa Frugoli***
}

\begin{abstract}
Resumo
O Transtorno de Escoriação é o ato repetitivo e compulsivo de provocar lesões na própria pele, sendo caracterizado como patológico quando é recorrente, o que causa desconforto e por diversas vezes gera sensação de culpa pela lesão, podendo gerar sofrimento psíquico significativo além de afetar direta e indiretamente a relação do indivíduo com si próprio e com o outro. O método para esta pesquisa foi a partir de uma revisão sistemática da literatura, com base na estratégia PRISMA, o que permitiu realizar uma coleta ampla e variada de diversos trabalhos realizados nesta temática, tanto na literatura nacional quanto internacional. Enquanto resultados, constatou-se que o transtorno possui maior predominância em mulheres, podendo também ser apresentado em indivíduos com outras comorbidades. Verificou-se também a necessidade de se refletir sobre este transtorno de um ponto de vista interdisciplinar, no qual a Psicologia pode contribuir significativamente com os aspectos subjetivos dos sujeitos acometidos por este transtorno. Palavras-chave: Transtorno de escoriação; Dermatilomania; Psicossomática; Psicologia.
\end{abstract}

\section{Skin Picking: Diagnosis and Intervention - A Systematic Review of Literature}

\begin{abstract}
Excoriation Disorder is the repetitive and compulsive act of causing injuries to the skin itself, being used as pathological when it is recurrent, or what causes discomfort and often causes the injury because of the injury, using the psychological suffering even more after the use and indirectly an individual's relationship with himself and with another. The method for this research was a systematic review of the literature, based on the PRISMA strategy, which made it possible to carry out a wide and varied collection of various works carried out on this theme, both in national and international literature. While the results, we found that the disorder is more prevalent in women, it can also be exhibited in other comorbidities. There was also a need to reflect on this disorder from an interdisciplinary point of view, no psychology can contribute to the subjective aspects of individuals affected by this disorder.

Keywords: Excoriation disorder; Skin Picking; Psychosomatic; Psychology.
\end{abstract}

\footnotetext{
* Psicólogo pela Universidade de Guarulhos e Mestre pelo PPGPS da UMESP

** Psicóloga pela Universidade de Guarulhos

*** Doutora em Saúde Coletiva pela UNIFESP e Docente do PPGPS da UMESP
} 


\section{Introdução}

Neste artigo buscou-se compreender o Transtorno de Escoriação (TE) por meio da formulação de conjecturas sobre as compreensões registradas na literatura, a partir das contribuições científicas a respeito desta temática e como esta transita entre as dimensões do ser humano, tendo em vista que a saúde mental se ramifica em uma vasta gama de segmentos que por sua vez disserta sobre os aspectos psicológicos que gravitam no entorno do processo do adoecer.

Ao olhar para o ser humano em integral, esta pesquisa dispõe de recursos reflexivos que e plurais que estabelecem uma ponte entre o orgânico e o psicológico, e em consecutivo, buscou-se elucidar quais os aparatos de diagnóstico, apoio, auxílio e tratamento presentes na comunidade de saúde para os indivíduos com este transtorno.

A fim de alcançar as proposições acima demarcadas, compreendeu-se que o Transtorno de Escoriação (TE), se dá a partir da aparição e frequência do comportamento repetitivo de lesionar a pele, promovendo um dano físico no tecido. Visto que além das implicações orgânicas, concomitantemente resulta em sofrimento psíquico, pois o indivíduo que apresenta TE percebe estar em um ciclo no qual inicialmente há o ferimento da pele, seguida por angústia/culpa pela lesão que se vincula a ânsia desmesurada que retoma a ocorrência do impulso e comportamento inicial (Coginotti \& Reis, 2016).

Enquanto transtorno, verifica-se que o TE é recente enquanto compreensões clínicas, uma vez que foi inserido no Manual de Diagnóstico e Estatístico de Transtornos Mentais - DSM em sua quinta (e mais recente) edição (2014), utilizando para seu enquadramento a terminologia “Transtorno de Escoriação (Skin-picking) (254)", sendo categorizado como 698.4 na especificação 235.

A partir de sua sintomatologia, o TE foi inserido no manual como sendo correlacionado com o "Transtorno Obsessivo-Compulsivo e Transtornos Relacionados", e tal relação se dá pelo padrão verificável de comportamentos repetitivos e rituais mentais que os transtornos desta terminologia apresentam, mesmo que existam caraterísticas distintas entre os demais quadros descritos nessa terminologia (Coginotti \& Reis, 2016).

O conteúdo abordado vem se evidenciando no decorrer dos últimos anos, porém sem muita suficiência para impulsionar um repertório mais volumoso, reiterado e expansivo de produções científicas, tanto em caráter documental, como em pesquisas de campo. Pretende-se que ao explorar o tema abordado pela antítese da visão que coloca o TE como sendo apenas uma questão de comportamentos conscientes, mas promover a compreensão de um condensado de eventos psíquicos e vivenciais que corroboram na intensificação deste comportamento, ascendendo ao status de transtorno (Coginotti \& Reis, 2016; Alves, Martelli, Prado \& Fonseca, 2009).

O Transtorno de Escoriação pode apresentar comorbidades concomitantes com outras psicopatologias, transtornos estes que também apresentam comportamentos autodestrutivos, auto lesivos, dificuldade em manifestar sentimentos de raiva, bem como sintomas do Transtorno Obsessivo Compulsivo, assim como ansiedade, depressão, transtorno dismórfico corporal, transtorno de personalidade borderline, tricotilomania, cleptomania, transtornos alimentares e transtorno de uso de substâncias (Alves et al, 2009).

Os comportamentos vinculados ao TE, podem trazer em si uma expressão simbólica do psíquico de tal indivíduo, pois a partir deste raciocínio, o transtorno comunica-se por meio do sintoma para evidenciar um desequilíbrio psicofisiológico, conduzindo assim o indivíduo rumo a integração constante de seus conteúdos psíquicos reprimidos, recalcados ou negados Alves et al, 2009; Müller \& Ramos, 2004).

Desta maneira, os processos psíquicos que não se elaboram por meio da comunicação verbal eclodem orgasticamente de maneira psicossomática, expressados então em conteúdos emocionais simbolizados, transportados em forma de comportamentos auto lesivos (Müller \& Ramos, 2004).

Em virtude do exposto, foi conduzido uma revisão bibliográfica de caráter sistemática em periódicos da área da saúde, objetivando alcançar relatos de casos e pesquisas aplicadas aos indivíduos com diagnóstico de TE, a fim de elencar as metodologias utilizadas no processo diagnóstico e no tratamento destes indivíduos e suas efetividades.

\section{Dermatilomania}

A partir de 1970, o ser humano passou a ser observado sob uma nova perspectiva, de forma que a não mais ser visto como fragmentado em suas facetas, mas como um indivíduo integrado, podendo ser compreendido em seus diversos aspectos. Esta nova visão reformula a compreensão de saúde existente na época, classificando então o ser humano como um ser indivisível. Seguindo essa linha de pensamento, o autor defende com base em sua compreensão, de que o indivíduo é biopsicossocial e espiritual e a importância equivalente destas esferas entre si na constituição do ser, com especial consideração aos 
seus aspectos comportamentais que constantemente são influenciados por seu corpo e mente (Ribeiro, 2011).

A pele por sua vez, possui parte ativa na integração do indivíduo, Oliveira (2018) relata que esta possui papel importante no exercício de vínculo do endógeno com o exógeno, pois além de ser o maior órgão humano, também comunica e recebe emoções e sensações do ambiente. O autor ainda afirma que a pele também traz uma representação de autoimagem, é por meio desta que a identidade do indivíduo se apresenta e torna-o singular, pois este órgão demonstra a ligação profunda existente entre ele mesmo e a mente humana.

De acordo com elucubrações realizadas por Silva e Muller (2007) verifica-se o reforço da ideia acima apresentada, visto que para os autores, a pele reflete todos os órgãos internos, fazendo um contato entre o interno e o externo do indivíduo. Portanto, quaisquer estímulos ou distúrbios apresentados na epiderme, serão refletidos no interior do corpo.

Tais construções de pensamento sugerem uma reflexão sobre o papel simbólico apresentado em distúrbios emocionais que possuem relação direta da autolesão com a pele, sendo assim, tais transtornos deixam evidentes mensagens contidas na alma e na psique, tendo em vista o papel ativo dela na imagem do sujeito (Feijoo e Mattar (2015).

A partir de pesquisa feita por Alves et al (2009) estima-se que entre 25 a 33\% dos casos dermatológicos possuem influência de uma disfunção psicossocial e raramente estes casos possuem correlação com problemas psiquiátricos.

A pele também exerce o papel ecológico, no qual o indivíduo utiliza a si mesmo como parâmetro de comparação, exercendo função de autoimagem, sendo o aspecto visível para com o outro, promovendo ou não autoconfiança servindo como parâmetro mediador entre o interno e o externo, permitindo a compreensão do self e promovendo a interação com o outro. Desta maneira, a pele se apresenta como campo de batalha entre o exógeno e o endógeno (Yoshinaga \& Galiás, 2018).

A pele traz em si a apresentação não apenas da situação em que nossos órgãos se encontram, como também demonstra os processos psíquicos e reações como a exteriorização das emoções. De maneira geral, a pele traspõe sentimentos involuntários como o enrubescer em situações de constrangimentos ou que evidenciam pensamento proibidos, de conteúdo sexual e até mesmo agressivo (Silva, 1994).

Por sua vez, a pele transpõe as ramificações do ser, se tornando o estágio final para a disseminação das emo- ções e exteriorização dos pensamentos e sentimentos. Ao passo que também é a janela que se abre para o toque em seus mais vastos significados. Cabe então, formular o que a dermatilomania traz em sua acepção mais profunda.

Ao refletir sobre a pele enquanto e suas funções subjetivas, Yoshinaga e Galiás (2018) propõem a ideia de que a pele manifesta na exterioridade aspectos sobre o 'ser' e os sentimentos das pessoas. Isso porque, a pele e a mente estão entre o "EU" psíquico/corporal e o "outro". Portanto, a pele é diferenciada do corpo e meio ambiente, bem como a psique diferencia o "EU psíquico" (nosso "mundo interior") do mundo externo. Ambos reforçam o conceito de que somos seres únicos e individuais. Sendo assim, a nossa pele é um órgão vital de comunicação. Para tanto, conceitos da psiconeuroimunologia têm buscado maior compreensão de como a pele e as nossas emoções se comunicam.

Sobre o fenômeno investigado nesta pesquisa, é importante o destacado por Pérez-Elizondo, Pino-Rojas e Gómez-Espinoza (2013), que informam sobre as escoriações, como consequências dos comportamentos de dilacerar a pele pelos quais o indivíduo manipula de maneira impulsiva ou compulsiva e até ritualística as imperfeições da pele, e com isso, causando lesões.

Sendo que os comportamentos auto lesivos ocorrem com maior frequência em locais de mais fácil acesso pelas mãos. Com isso, além dos danos na pele ocorre também a desfiguração da autoimagem do indivíduo, o que pode alterar seu desempenho psicossocial. Isso porque, este possui pouco controle dos impulsos, podendo se tratar de um sintoma de um transtorno obsessivo-compulsivo com traços de ansiedade e componente depressivo (Alves et al, 2009).

Por meio de estudos sociodemográficos, Garreto (2015) afirma que os comportamentos de automutilação são mais propensos inicialmente na fase da adolescência, onde o indivíduo possui dificuldade no manejo de seus afetos e na resolução dos problemas, já que estes possuem maiores limitações cognitivas devido a seu processo de desenvolvimento, pois é justamente neste período em que os adolescentes começam a identificar e vivenciar as emoções de forma mais intensa, ao passo que também estão desenvolvendo a capacidade de cognição social e, portanto, as estruturas cerebrais que auxiliariam nessa expressão ainda não estão desenvolvidas.

O mesmo autor anexa que $90 \%$ dos indivíduos que apresentam esses comportamentos na adolescência, não os mantêm em sua fase adulta. Tal informação elucida sobre a necessidade existente de avaliação prévia para 
maior compreensão dos quadros apresentados, viabilizando então projetos de prevenção de tais comportamentos, manutenção da saúde mental, assim como intervenções em casos de aparecimento do comportamento. Contudo, até o presente momento não existem estudos nacionais publicados a respeito da probabilidade e frequência da automutilação em quaisquer faixas etárias.

A dermatilomania é diagnosticada em sucessão a consciência da provocação dos comportamentos auto lesivos e da confissão verbal do paciente de ser o autor das lesões em sua pele, e para tal diagnóstico se faz necessário a exclusão de todas as demais possibilidades de transtornos previamente, conforme relata (Alves et al, 2009).

Comorbidades psiquiátricas em pacientes com skin picking são comuns, principalmente no que se refere a transtornos de humor e ansiedade. Essas, podem se tratar de transtorno obsessivo-compulsivo, transtorno dismórfico corporal, transtornos por uso de substâncias, distúrbios alimentares, tricotilomania, cleptomania, comprar compulsivo, transtorno de personalidade obsessivo-compulsivo e transtorno de personalidade borderline. De forma que a distorção da aparência por sua vez, interfere na vida pessoal, social e no trabalho do indivíduo (Domingues, Alves, Colenghi, Lima, Pinheiro, Paes \& Silva 2017).

A progresso dos quadros de comorbidades psiquiátricas como o transtorno obsessivo compulsivo assim como verificado em outros transtornos ansiosos, se apresenta após o uso contínuo de no mínimo três a doze meses de tratamento medicamentoso e acompanhamento psicológico, pois estes corroboram para a melhora do humor da ansiedade bem como melhoras nos sintomas depressivos. Engloba-se também dentro dos transtornos do espectro obsessivo compulsivo a dermatilomania pois nesta também foi constatada uma significativa evolução dos sintomas compulsivos (Valerio, 2011).

Segundo resultados encontrados na amostra de Giusti (2013), adultos brasileiros que praticam a automutilação também buscam tratamento num serviço terciário de saúde assim como nos pacientes registrados nos estudos internacionais. Esses se assemelham na idade, tipos de mutilação e motivações, de forma que os dados apresentados nos estudos internacionais se tornam validos para a realidade apresentada nos estudos nacionais.

Em virtude dos estudos elaborados a respeito das características sociodemográficas com base no panorama das comorbidades associadas ao transtorno de controle dos impulsos, Domingues et al, (2017) relata que a dermatilomania (Skin Picking) foi o transtorno com maior nú- mero de diagnósticos simultâneos presente nas amostras, reforçando a ideia de que a tricotilomania e a dermatilomania compõem um subgrupo específico de transtornos associados aos comportamentos de autocuidado.

\subsection{Psicossomática}

Com o intuito de buscar contribuições teóricas do já consolidado campo da psicossomática, Campos e Rodrigues (2005, p.292), apontam que a mesma consiste no "estudo sistemático das relações existentes entre os processos sociais, psíquicos e transtornos de funções orgânicas ou corporais."

$\mathrm{Na}$ qualidade dialógica sobre os aspectos da psicossomática, Capitão e Carvalho (2006) afirmam que a dor e o conflito psíquico no indivíduo gerados por fatores de estresse podem culminar na sua incapacidade de tolerância, atrapalhando então seu processo de reconhecimento, simbolização e elaboração dos mesmos. Sendo assim, o estresse pode ser descarregado por meio de manifestações somáticas, e o adoecimento se torna uma maneira de estabelecer o equilíbrio para o corpo.

De acordo com Valente (2012), no que se refere à psicossomática, compreende-se que as obras de Freud e Alexander são as principais referências psicanalíticas acerca do tema. Para o autor, estes defendem a ideia de que este fenômeno se origina na dificuldade de simbolização das emoções do sujeito frente às transmutações ao decorrer da vida. Isso porque, quando o psiquismo não possui recursos para lidar com determinada situação, a emoção atrelada a esse fenômeno permanece no corpo, aumentando a vulnerabilidade do indivíduo e passando a produzir sintomas somáticos.

Tal fenômeno é nomeado por Bion (1991) como Sistema protomental, no qual o psíquico e o físico se diferenciam, o que ratifica a hipótese da expressão aparente no plano de situações provedoras de angústia/aflição, que pode se tornar evidente de ambas as formas. Esta conjectura possui correlação com a compreensão vinculada à formação inicial do corpo junto a mente, quando estes ainda encontram se indiferenciados:

Dois com um corpo: relata fantasia primordial, presente em todo ser humano, visa fazer um, com a mãe-universo da pequena infância. [...] A partir dessa matriz somatopsíquica, uma diferenciação progressiva entre o corpo próprio e a primeira representação do mundo externo, que é o seio materno, vai se desenvolver na psique infantil. Paralelamente, o que é psíquico vai se distinguindo, aos poucos, do que é somática (McDougall, 1987 como citado em Ávila, 2016). 


\subsection{Psicodermatologia}

A relação da psicodermatologia e a medicina psicocutânea vêm ganhando ênfase ao longo dos anos por ambas tratarem da relação entre as doenças mentais com as doenças da pele, de forma em que interligam duas especialidades médicas: psiquiatria e dermatologia. A primeira visando compreender os aspectos internos na psique do indivíduo, enquanto a segunda diagnostica e trata as doenças de pele, sendo esses, aspectos externos. Para tanto, o profissional da saúde precisa correlacionar essas duas especialidades e analisar a comunicação dos aspectos biológicos e psicológicos que as norteiam. (Ávila, 2016).

A história natural do indivíduo e as implicações da desordem específica de pele são fatores que possuem impacto no que se refere ao impacto das doenças cutâneas. Logo, a análise de características demográficas, de personalidade, caráter e valores do paciente, sua situação de vida e comportamentos sociais sobre o significado dos distúrbios de pele se fazem necessárias. (Ginsburg \& Link, 1993).

Os fenômenos de alta complexidade têm chamado a atenção das áreas da saúde, e estas por sua vez têm se dedicado a busca por tais conhecimentos, pois a necessidade de corroborar com produções de caráter interdisciplinar tem se evidenciado constantemente.

A fim de sanar tais necessidades, surgiu a Dermatologia Integrativa com eixo na Psiconeuroimunologia, estas construções promovem a compreensão da mutualidade da mente e corpo do indivíduo e a condução de aparatos que auxiliem na diminuição do estresse e maior ganho no tratamento (Alves et al, 2009).

O mesmo autor ainda ressalta o vínculo multiprofissional descrito pela Psicodermatologia, onde psicólogos e dermatologistas unem esforços no desenvolvimento de um trabalho que seja contínuo e interligado, propondo uma integração de conhecimentos específicos e a promoção de pesquisas e tratamentos de dermatoses que são classificadas como tendo fundo fenomenológico de caráter psíquico.

No toante à sintomatologia expressiva dos indivíduos diagnosticados com Transtorno de escoriação Freitas (2011), aponta que estes pacientes podem apresentar comportamentos compulsivos em decorrência de vivências de angústia, ansiedade e solidão. Estes também podem manifestar com demasiada frequência, sentimento de culpa e inferioridade além de conduta compulsiva em suas atividades, se equiparando a persistência com que realizam as escoriações.
Eventualmente podem ocorrer equívocos diagnósticos em virtude das semelhanças existentes entre o transtorno de escoriação e a dermatite, sendo que estas falhas ocorrem principalmente em casos nos quais o sujeito por ausência de recursos psíquicos, não obtém consciência de seu papel ativo na autoria das lesões, omitindo assim tais fatos dos profissionais de saúde que estão acompanhando seu caso (Ribeiro, Ribeiro \& Doellinger, 2015).

Em algumas situações, podem ocorrer erros nos diagnósticos clínicos, visto que o transtorno de escoriação pode se confundir com a dermatite, quando o paciente não assume a autoria da lesão. Isso porque, devido à falta de recursos psíquicos o paciente não obtenha consciência do fato, omitindo assim informações que fizeram parte do processo de lesão (Ribeiro, Ribeiro \& Doellinger, 2015).

Com base no postulado por Alves, Martelli, Prado e Fonseca (2009) pode-se notar que uma gama representativa destes pacientes possui traumas emocionais ocorridos na infância, adolescência, provocado dificuldades no desenvolvimento infantil, desadaptação e consequentemente na vida adulta apresentam ausência de Recursos de enfrentamento diante das situações da vida, imaturidade psicológica na dificuldade na eficácia do controle de impulsos.

Sobre a complexidade subjetiva presente neste cenário, Freitas (2011), ressalta que indivíduos com tais características apresentam dificuldades intrínsecas no trato em suas relações pessoas, pois possuem dificuldades no delineamento de vínculos afetivos, apresentando por vezes comportamentos controladores em suas relações, pois se apoiam emocionalmente na relação com o outro, promovendo conflitos interpessoais.

Logo, verifica-se que indivíduo com este diagnóstico denota características de extrema dificuldade em delinear seus vínculos afetivos, buscando com frequência apoio emocional no outro, por vezes até tendenciado a controlar o comportamento de seus companheiros gerando conflitos em suas relações por seu direcionamento emocional em relação as questões da vida (Freitas 2011).

Perseguindo os sintomas auto lesivos, cabe apontar que estes são reforçados em decorrência dos sentimentos de repulsa vivenciados por pessoas com a pele escoriada, pois os ferimentos proporcionam um grau de comprometimento da aparência, podendo variar de leve a grave, interferindo na autopercepção de tais indivíduos.

Estes sentimentos possuem influência direta nos prejuízos apresentados pelos indivíduos em suas relações afetivas e profissionais, podendo promover em casos de maior gravidade empecilhos em situações rotineiras como: 
realização de higiene pessoal no momento do banho, se olhar no espelho além de se vestir (Jorge, Müller, Ferreira \& Cassal, 2004).

Em harmonia com os relatos, Lovato (2011) conjectura que diversos casos de indivíduos com TE não obtiveram diagnostico em virtude da ausência de compreensão do Transtorno ou encontram-se sub-diagnosticados, transpõe também que a escoriação psicogênica pode ser considerada como um fenômeno raro se vista de maneira isolada na prática clínica, porém com a ramificação de informação a respeito da temática e se aglomerarmos os casos, estes apresentam uma quantidade expressiva.

\section{Método}

O presente estudo utilizou da enquanto ferramenta metodológica a revisão sistemática da literatura, conduzido conforme as diretrizes do Preferred Reporting Items for Systematic Reviews and Meta-Analyses - PRISMA (Moher, Shamseer, Clarke, Ghersi, Liberati, Petticrew, Shekelle, Stewart \& PRISMA-P Group, 2015). Este tipo de revisão caracteriza-se como um estudo secundário que visa angariar dados específicos sobre as indagações científicas realizadas pelas pesquisadoras.

A pesquisa foi conduzida por duas pesquisadoras e, para garantir a rigidez do processo de coleta de dados, contou com a revisão de dois supervisores.

No que diz respeito a operacionalização da pesquisa, inicialmente, foram estabelecidos descritores para possibilitar a localização de artigos relevantes para a pesquisa. Para isto recorreu-se ao vocabulário de terminologias indexado ao DeCS (Descritores em Ciências estes da Saúde). Com estes localizados, foram organizados da seguinte maneira para a busca: "Transtorno de Escoriação"; "Dermatilomania"; "Skin Picking"; "Psicossomática"; "Psicodermatologia" e "Psicodermatologia AND Saúde mental". Esta busca se deu nas bases de dados Lilacs, Scientific Electronic Library Online (SCIELO), Periódicos Capes, Biblioteca Virtual em Saúde (BVS) e Biblioteca Digital de Teses e Dissertações da USP.

O levantamento dos dados bibliográficos ocorreu de fevereiro a junho de 2019 com base nos critérios de inclusão estabelecidos. Estes seguiram as seguintes considerações: (a) teses, artigos narrativos, cujos temas abordavam as sintaxes definidas (b) teses, artigos narrativos, artigos de revisão sistemática que correlacionassem a psicologia com os temas presentes nos descritores; (b) títulos dos quais os objetivos obtivessem compatibilidade com a área da saúde; (c) artigos publicados em idiomas inglês, espanhol e português.

A partir dos resultados obtidos inicialmente, foram propostos critérios de exclusão: (a) artigos/teses cuja publicação ultrapassava o período de dez anos; (b) artigos/teses em duplicidade; (c) formatos de textos que não eram científicos; (d) produções que não passaram por processo rigoroso de avaliação (d) títulos divergentes ao tema; (e) áreas do conhecimento que fossem incompatíveis com a saúde; (f) artigos/teses em idiomas diferentes aos estipulados na pesquisa; $(\mathrm{g})$ artigos/teses cujas fontes não se obteve acesso.

Posteriormente foi realizada a seleção mediante a leitura e a análise crítica dos títulos e resumos de todos os artigos identificados. Em seguida, foi feita uma leitura na íntegra dos estudos selecionados, a qual possibilitou que outros textos também fossem excluídos por não atenderem à proposta da revisão.

Por fim, as principais informações dos artigos foram sintetizadas em uma planilha para que pudessem orientar as análises posteriores.

Os Artigos selecionados para a composição da amostra foram divididos em duas categorias, a primeira apontando dados sociodemográficos e recursos disponíveis mencionados nos artigos como auxílio ao indivíduo com transtorno de escoriação, e a segunda indicando os aspectos psicossomatológicos e fenomenológicos do transtorno de escoriação, demonstrando as abordagens utilizadas pelos autores bem como alternativas dos possíveis tratamentos de forma a acrescentar às pesquisas realizadas.

\section{Resultados}

De forma geral, foram utilizados como parâmetro os 12 artigos delimitados, conforme a Tabela 2. Classificados em caráter longitudinal, sendo apresentados da data de publicação mais distante ao produzido mais recentemente, estes foram classificados como resultantes da revisão sistemática realizada previamente.

Tendo sido utilizados 7 artigos produzidos no Brasil em sua gama diversificada de estados, sendo estes dos estados de São Paulo (4), Rio de Janeiro (2) Fortaleza (1), foram também utilizados 4 artigos dos EUA publicados em Chicago (2), Vale do Moreno (1) e (1) artigo publicado em estado indefinido, além de 1 artigo Publicado em Buenos Aires - Argentina e (1) em Diyarbakir, Turquia. 
TRANSTORNO DE ESCORIAÇÃO: DIAGNÓSTICO E INTERVENÇÃO - UMA REVISÃO SISTEMÁTICA

Tabela 1 - Protocolo Prisma

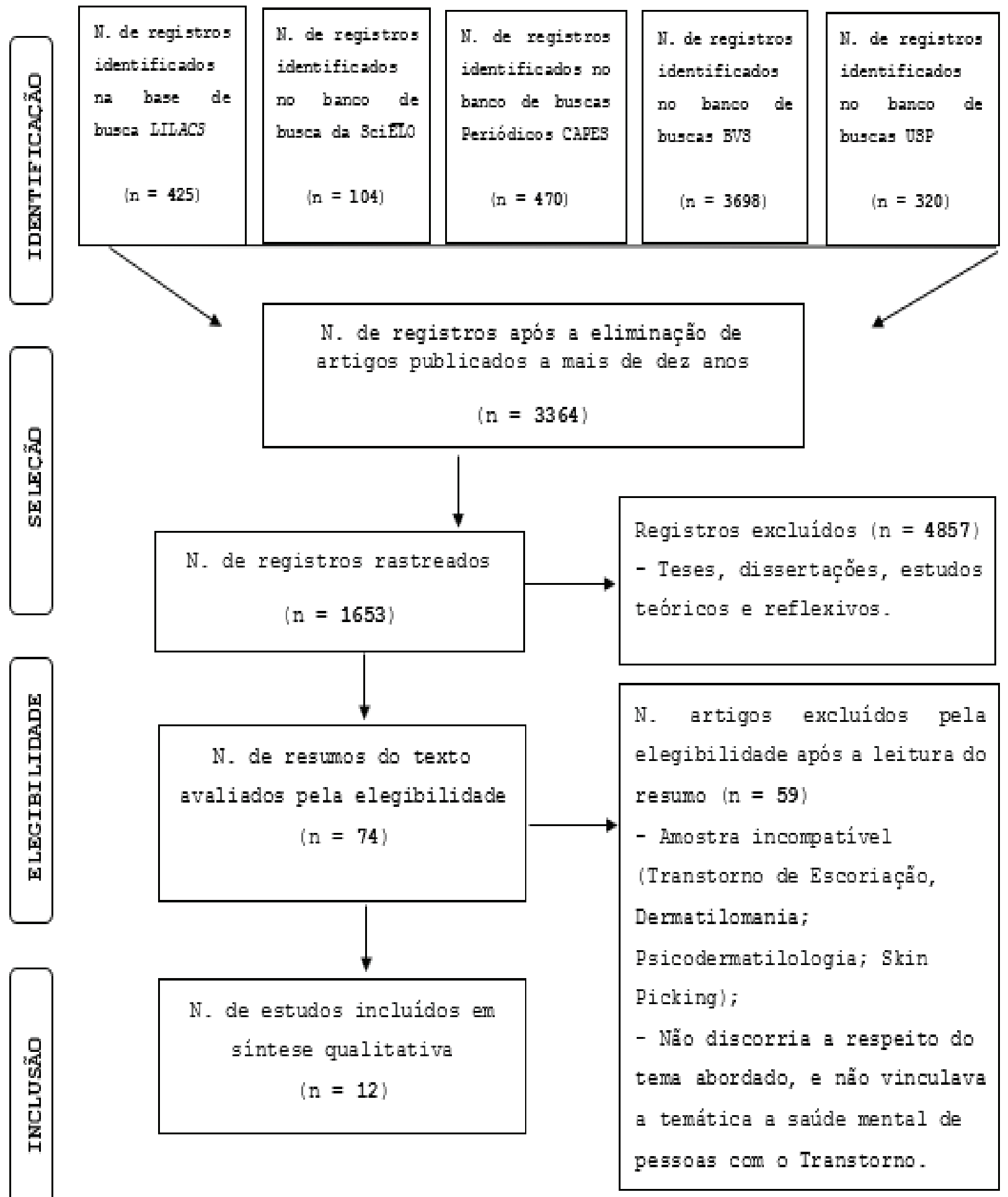

Fonte: Elaborado pelas autoras 
Tabela 2 - Descritivo de Artigo/Teses resultantes em consecutivo a revisão sistemática.

\begin{tabular}{|c|c|c|c|}
\hline NOME DO ARTIGO & ANO & PAISI ESTADO & AUTOR \\
\hline $\begin{array}{l}\text { Variabilidade de diagnósticos psicológicos } \\
\text { frente à avaliação dermatológica da escorią̧ão } \\
\text { psicogênica }\end{array}$ & 2009 & $\begin{array}{l}\text { Brasil, Rio de } \\
\text { Janeiro. }\end{array}$ & $\begin{array}{l}\text { ALVES,C.J.M.:MARTELLI, A.C. } \\
\text { C.; PRADO, R. B. R.; FONSECA, M.S. }\end{array}$ \\
\hline $\begin{array}{l}\text { O corpo sem mor ada: a doença } \\
\text { psicossomática como expressão do } \\
\text { desfundamento da pessoa humana na } \\
\text { sociedade contemporânea }\end{array}$ & 2010 & Brasil, Fortaleza. & MAIA, M. V.M.; PINHEIRO, N. N. B. \\
\hline Skin picking disorder & 2012 & Estados Unidos & $\begin{array}{l}\text { GRANT, J.E; ODLAUG, B. L.; } \\
\text { CHAMBERLAIN, S.R.; KEUTHEN, N. } \\
\text { J.:LOCHNER, C. L.STEIN, D. J. }\end{array}$ \\
\hline $\begin{array}{l}\text { Excoriaciones psicogénic as: identificación, } \\
\text { interpretación y abordaje de una patología de } \\
\text { atención compartida }\end{array}$ & & $\begin{array}{l}\text { Argentina, Buenos } \\
\text { Aires. }\end{array}$ & $\begin{array}{l}\text { ELISONDO, A.D.P.; ROJAS, G. T.P.; } \\
\text { ESPINOZA, M. G. }\end{array}$ \\
\hline $\begin{array}{l}\text { A atuação da equipe multiprofissional } \\
\text { nodiagnóstico e tratamento da escoriação } \\
\text { psicogênica }\end{array}$ & 2013 & Brasil, São Paulo. & $\begin{array}{l}\text { STEVANATO, D; PRADO, R.B.R: } \\
\text { MARTELLI, A.C.C. }\end{array}$ \\
\hline A near fatal case of pathological skin picking & & $\begin{array}{l}\text { Estados Unidos, } \\
\text { Vale de Moreno. }\end{array}$ & $\begin{array}{l}\text { KIM, D.S; GA.RISSON, R.C.: } \\
\text { THOMPSON, G. }\end{array}$ \\
\hline $\begin{array}{l}\text { Automutilação: características clínicas e } \\
\text { comparação com pacientes com transtorno } \\
\text { obsessivo-compulsivo }\end{array}$ & & Brasil-São Paulo. & GIUSTI, J.S. \\
\hline $\begin{array}{l}\text { Body-focused repetitive behavior disorders in } \\
\text { ICD-11 }\end{array}$ & 2014 & Chicago, EUA. & GRANT, J. E.; STEIN, D. J. \\
\hline $\begin{array}{l}\text { A skin-picking disorder case report: a } \\
\text { psychopathological explanation }\end{array}$ & 2015 & $\begin{array}{l}\text { Brasil, Rio de } \\
\text { Janeiro. }\end{array}$ & $\begin{array}{l}\text { RIBEIRO, \&.; RIBEIRO, J.P: } \\
\text { DOELLINGER, O.V. }\end{array}$ \\
\hline $\begin{array}{l}\text { O desempenho executivo em pacientes que } \\
\text { apresentam automutilação }\end{array}$ & & Brasil, São Paulo. & GARRETO, A.K.R. \\
\hline Genital Dermatillomania & 2017 & Chicago, USA. & $\begin{array}{l}\text { ALEXANDROV.P.A.:TAN, W.P. } \\
\text { B.; ELTERMAN, L.B. }\end{array}$ \\
\hline $\begin{array}{l}\text { Os aspectos psicopatológicos e } \\
\text { fenomenológicos do transtorno de escoriação }\end{array}$ & 2018 & Brasil, São Paulo. & OLIVEIRA,E.C.B. \\
\hline
\end{tabular}

Categoria 1 - Alternativas de Tratamentos ao indivíduo com transtorno de escoriação.

Tabela 3 - Artigos que compõe a categoria 1.

\begin{tabular}{|c|c|c|c|c|c|c|}
\hline NOME DO ARTIGO & País & $\begin{array}{l}\text { HOUVE } \\
\text { AMOSTRA }\end{array}$ & $\begin{array}{l}\text { ABORDAGEM } \\
\text { UTILIZADA }\end{array}$ & $\begin{array}{l}\text { ALTERNARIVAS DE } \\
\text { TRATAMENTOS }\end{array}$ & AUTOR & ANO \\
\hline $\begin{array}{l}\text { Variabilidade de diagnósticos } \\
\text { psicológioos frente à avaliaç5̆o } \\
\text { dermatológioa da escoriaçăo } \\
\text { psicogênica }\end{array}$ & Brasil & Sim & $\begin{array}{l}\text { Psicoterapia } \\
\text { comportamental, } \\
\text { focal ou breve }\end{array}$ & $\begin{array}{l}\text { Antidepressivos; } \\
\text { Antipsicóticos; } \\
\text { Psicoterapia. }\end{array}$ & $\begin{array}{l}\text { ALVES, C. J.M.; MARTELLI, } \\
\text { A. C. C.; PRADO, R. B. R; } \\
\text { FONSECA,M.S. }\end{array}$ & 2009 \\
\hline Skin pioking disorder. & $\begin{array}{l}\text { Estados } \\
\text { Unidos }\end{array}$ & Sim & $\begin{array}{l}\text { Terapia Cognitivo } \\
\text { Comportamental }\end{array}$ & $\begin{array}{l}\text { Psiquiatra:Dermatologist } \\
\text { a. Terapia Cognitivo } \\
\text { Comportamental. }\end{array}$ & $\begin{array}{l}\text { GRANT, J.E.; ODLAUG, B. } \\
\text { L.; CHAMBERLAIN, S.R.; } \\
\text { KEUTHEN, N. J.; LOCHNER, } \\
\text { C. L.; STEIN, D. J. }\end{array}$ & 2012 \\
\hline $\begin{array}{l}\text { A ATUACAOAODA EQUIPE } \\
\text { MULTPROFISSIONAL NO } \\
\text { DIAGNOSTICOE TRATAMENTO } \\
\text { DA ESCORIAÇAOOPSICOGENICA }\end{array}$ & Brasil & Nรั่ & Psicanálise & $\begin{array}{l}\text { Psicoterapia; } \\
\text { antidepressivos } \\
\text { tricíclicos. }\end{array}$ & $\begin{array}{l}\text { STEVANATO, D:PRADO, } \\
\text { R.B.R.:MARTELLI,A.C.C. }\end{array}$ & \\
\hline $\begin{array}{l}\text { Excoriaciones psicogénicas: } \\
\text { identifioación, interpretacióny } \\
\text { abordaje de una patología de } \\
\text { atención compartida }\end{array}$ & Argentina & Nड̃̀o & Não evidenciada & $\begin{array}{l}\text { Dermatologix } \\
\text { F́́rmacos. }\end{array}$ & $\begin{array}{l}\text { ELISONDO, A. D. P.: } \\
\text { ROJAS, G. T.P.: } \\
\text { ESPINOZA,M. G. }\end{array}$ & 2013 \\
\hline $\begin{array}{l}\text { A near fatal case of } \\
\text { pathological skin } \\
\text { picking }\end{array}$ & $\begin{array}{l}\text { Chicago, } \\
\text { suA. }\end{array}$ & Sim & Não evidenciada & $\begin{array}{l}\text { Psiooterapia; } \\
\text { Farmacoterapia. }\end{array}$ & $\begin{array}{l}\text { KIM, D.S.; } \\
\text { GARISSON, R. C.; } \\
\text { THOMPSON, G. }\end{array}$ & \\
\hline Genital Dermatilomania & $\begin{array}{l}\text { Estados } \\
\text { Unidos }\end{array}$ & Sim & N5o evidenciada & $\begin{array}{c}\text { Tetapis } \\
\text { comportamental; } \\
\text { Medicamentos. }\end{array}$ & $\begin{array}{l}\text { ALEXANORON,P. A.; TAN, } \\
\text { W.P. B.; ELTERMAN, L. B. }\end{array}$ & 2017 \\
\hline
\end{tabular}


Dos 6 artigos estabelecidos para esta categoria (Tabela 2). Evidenciam (Alves, Martelli, Prado \& Fonseca, 2009; Grant \& Stein, 2014; Stevenato, Prado \& Martelli, 2013; Elizondo, Rojas \& Espinoza, 2013; Kim, Garrison \& Thompson, 2013 e Alexandrov Tan, \& Elterman, 2017) que o Transtorno de Escoriação possui como melhor alternativa de tratamento o trabalho multidisciplinar, sendo que é indicado ao indivíduo diagnosticado a intervenções medicamentosas atrelada a psicoterapia, para tal Grant e Stern (2013), apenas indica o acompanhamento de um Psiquiatra no decurso do Tratamento, já Stevenato, Prado \& Martelli (2013), indicam os antidepressivos tricíclicos como sendo de maior eficácia.

Demais autores citados, reforçam a importância do tratamento medicamentoso, mas que a utilização destes medicamentos, assim como sua dosagem variam de acordo com os casos, e portanto ressaltam a importância do diagnóstico precoce da dermatilomania dentre os profissionais de saúde envolvidos, para que assim possam fazer trabalhos multidisciplinares, tanto pelas potenciais complicações clínicas quanto pelo prejuízo psicossocial do indivíduo.

Para auxílio dessa esfera, os autores indicam a psicoterapia como necessária para evolução do quadro, para tal Alves, Martelli, Prado e Fonseca (2009); Stevenato, Prado e Martelli; Pérez-Elizondo, Pino-Rojas e Gómez-Espinoza (2013) e Kim, Garrison e Thompson, (2013); indicam a Psicoterapia como necessária, já Grant e Stern(2012) e Alexandrov Tan e Elterman, (2017), indicam a Terapia comportamental e Cognitivo comportamento como sendo a mais indicada para a evolução do quadro clinico dos pacientes com tal transtorno. Em todos os artigos citados, os autores ressaltam que as consequências de tal transtorno podem afetar o desenvolvimento desde a infância até a vida adulta, sendo de início mais tardio, muitas vezes de evolução crônica, potencialmente grave e geradora de sofrimento e impacto relevantes.

\section{Categoria 2: Aspectos psicossomatológicos e fenomenológicos do transtorno de escoriação.}

Para elucidação da Tabela 4 cabe ressaltar que ao descrever Aspectos Psicossomatológicos os autores evidenciam a descrição do quadro clínico físico e psicossomático do enfoque apresentado pelo paciente.

De forma geral, os dados encontrados no estudo de Giusti (2013); discorre sobre o perfil sintomatológico e características clínicas partilhadas a partir do transtorno, como as escoriações apresentadas na pele do sujeito, assim como localização destas e a sintomatologia correlacionada as lesões e as medidas Interdisciplinares indicadas pelos profissionais para correção dos danos, pois a pele é considerada o maior órgão do corpo humano.

Quando correlacionamos estes dados compreendemos que os aspectos sintomatológicos possuem direta associação com os processos psicológicos. De forma que a pele quando lesionada, leva a sofrimento emocional e a afastamento afetivo e social.

Este está vinculado aos Aspectos Fenomenológicos: que descrevem a gênese do sintoma, além da apresentação por intermédio de discussão de casos da percepção do indivíduo frente a estes. Os estudos de Giusti, (2013); Garreto, (2015); İbiloglu, Abdullah, Kaya, Demir, Bulut e Sir, (2016) e Oliveira, (2018). Evidenciam a Gênese do sintoma e não apenas aspectos físicos proporcionados em virtude de tais processos desencadeadores, tal como os fatores desencadeantes como a dificuldade em lidar

Tabela 4 - Artigos que compõe a categoria

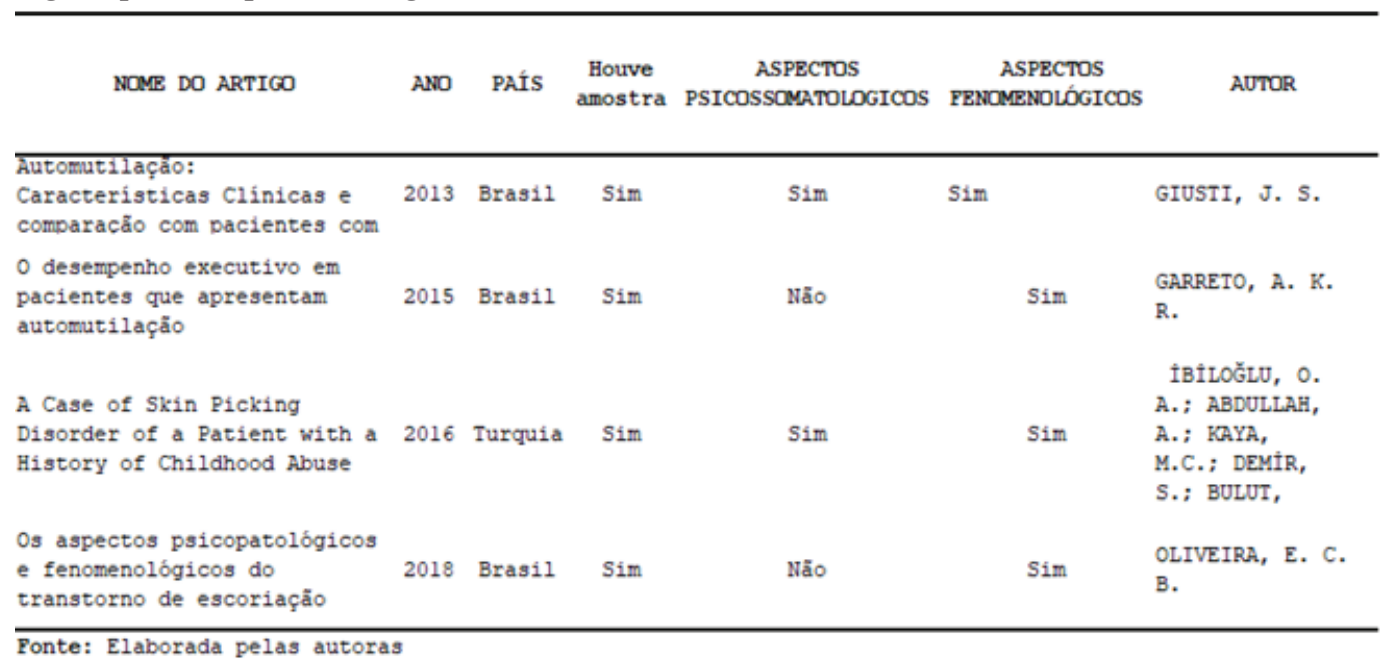


com os próprios impulsos e a compulsão, pois o paciente admite ser o causador das lesões, porém, de maneira involuntária. Tais estudos investigaram a história de vida, os aspectos psicológicos e os fatores de personalidade possivelmente relacionados à escoriação psicogênica dos indivíduos diagnosticados.

\section{Discussão}

O transtorno de escoriação apresenta relevante importância diante da área da saúde, visto que ainda são avaliadas quais as abordagens e tratamentos se fazem necessários diante da variabilidade dos casos, bem como sua eficácia. Isso porque, dentro do campo científico, denota-se escassez no número de pesquisas realizadas no campo da psicologia e psiquiatria a respeito do tema.

Isso torna-se claramente verificado em primeiro momento, quando obteve-se apenas resultados no DeCS a partir da busca por "psicossomática" diante dos descritores. Posteriormente, foram rastreados resultados com as terminologias Dermatotilexomania1 e Desordem cutânea.

A partir da revisão bibliográfica realizada, aspectos relacionados à conjuntura atual das pesquisas devem ser discutidos. Este fato, somado aos resultados, representa uma fonte de pesquisa para estudos posteriores, tanto na investigação de diretrizes para avaliação diagnóstica quanto para as alternativas de tratamento.

Segundo análises, compreender tais dificuldades a extensão dessas pesquisas é crucial para diagnosticar e tratar pacientes, à medida que maiores produções científicas podem trazer inovação no que se refere ao processo de diagnóstico e intervenções. Estudos futuros são necessários para investigar também a predisposição de alguns pacientes em relação ao transtorno.

Além disso, os resultados obtidos a partir do nosso estudo demonstram claramente que essas informações afirmam que a promoção do tema se faz urgente à população geral, também como meio de prevenção.

Destaca-se a importância de evidenciar a conformidade dos pesquisadores diante do tema na sugestão como forma de tratamento unificado um trabalho multidisciplinar, interligando os profissionais da saúde, constituído por psicólogos, psiquiatras e dermatologistas.

De acordo com as amostras, também há conformidades mencionadas nos artigos analisados, visto que essas apontam concordância em relação às intervenções, sendo composta pela interposição medicamentosa aliada as diferentes vertentes da psicoterapia, podendo ser utilizada a Terapia breve concomitante a Terapia Cognitivo - Comportamental ou Psicanálise.
Entretanto, ainda enquanto ao tratamento, observa-se oposição nas perspectivas dos autores, visto que não há conformidade nas abordagens psicológicas propostas, de forma que as opções de tratamento se mostram vagas. Este fato somado a poucas terapias alternativas salienta deficiência a respeito do tratamento, uma vez que a diversificação de novas terapias tem tido relevante aumento ao decorrer dos anos.

De forma geral, a terapêutica deve considerar as particularidades de cada paciente, sendo que, para sua definição, é imprescindível a realização de estudos de casos clínicos, em equipe interdisciplinar.

Dentre os estudos acerca dos aspectos psicossomatológicos apenas metade dos autores atribui esse fator como relevante em suas amostragens. Ao passo que os fatores fenomenológicos foram propostos de forma unânime dentre as literaturas.

As análises de pesquisas também revelam a prevalência nos resultados em relação ao gênero feminino, visto que a incidência do transtorno no gênero masculino é consideravelmente menor.

O estudo conduzido por Gomes, Ferreira do Nascimento e Araújo (2007) aponta que o ideal configurado pelo homem, arraigado a fatores culturais é um obstáculo na adoção de práticas de autocuidado, visto que o homem é visto como forte e viril e, portanto, o ato de buscar serviços de manutenção da saúde poderia associá-lo à fraqueza, medo e insegurança. Complementam também, que por conta dessa masculinidade instituída, este ato poderia aproximar homens das representações do universo feminino, o que implicaria possivelmente desconfianças acerca de sua sexualidade.

A performatividade masculina na sociedade ocasionalmente desdenha de questões tidas como "domésticas", inclusive o autocuidado (Saffioti, 1987).

Diante disso, pode-se correlacionar estudos científicos com as amostras desta pesquisa, pois essas também entendem que as relações de gênero são relevantes e afetam as percepções dos indivíduos em relação a si próprios.

De modo geral, salienta-se que é de extrema relevância enfatizar que muito ainda pode ser investigado acerca do transtorno de escoriação. Recomenda-se que futuras pesquisas possam enfocar a prevenção, diagnóstico, proposta de tratamentos e intervenções, fazendo a correlação da saúde mental e dermatológica. Estudos em maiores escalas também se fazem necessários, a fim de contribuírem com maiores dados sobre o tema para que este alcance maior notoriedade. 
Torna-se indispensável que uma participação informada e ativa por parte dos profissionais de psicologia para a disseminação do tema, de forma a produzir pesquisas relevantes com maiores dados sobre esse tema que não possui a visibilidade necessária.

\section{Considerações Finais}

O estudo em questão buscou compendiar de forma elucidativa compreensões acerca do transtorno de escoriação e suas dialógicas com aspectos psicossomáticos e fenomenológicos.

É importante destacar também, que foram coletados dados a partir de uma revisão sistemática, da qual foram identificados e analisados estudos nacionais e internacionais, de acordo com a proposta e os critérios estabelecidos, o que possibilitou o encontro de possibilidades para compreender e refletir sobre esse transtorno.

Neste sentido, cabe refletir no que se refere à quantidade de produções científica obtidas em território nacional e internacional e preponderar o seu caráter e extensão. Vale reiterar que apesar de terem sido utilizadas publicações nacionais em maior número, isto ainda denota uma lacuna no conhecimento científico sobre o TE, tornando precário o diagnóstico, bem como as intervenções quanto ao transtorno.

Esta pesquisa serviu também para verificar uma correlação entre a dermatilomania e outros transtornos psiquiátricos vinculados à pele, por estarem associados à dificuldade de controle de impulsos e aspectos obsessivos da personalidade, entretanto cabe situar que existe variância entre níveis e estágios diferentes entre os pacientes.

Acredita-se que também que os estudos na área têm sido de suma importância para calcar terreno fértil para novas pesquisas que coloquem em relevo aspectos para além dos clínicos, mas aglutinando em seu bojo compreensivo fatores culturais e sociais, que os artigos aqui utilizados destacam de forma expressiva. Enfatiza-se a importância de estudos mais enfocados e extensos, com maiores amostras heterogêneas para a investigação e compreensão destes eventos.

Por fim, ao considerar os danos na qualidade de vida dos pacientes acometidos com o transtorno de escoriação, é importante humanizar o processo médico a partir do trabalho psicológico, visto que os dermatologistas devem considerar sempre os danos psicológicos dentro do processo de diagnóstico. Tanto a avaliação quanto o acompanhamento psicológico se fazem indispensáveis.

\section{Referências}

Alexandrov, P.; Tan, W, P. \& Elterman, L. (2017, november) Genital Dermatillomania. Current Urilogy 11,54-56. doi: https//doi.org/10.1159/000447195

Alves, M. J. C., Martelli, C. C. A., Prado, R. B. R. \& Fonseca, R. M. (2009, setembro/ outubro) Variabilidade de Diagnósticos Psicológicos Frente à Avaliação Dermatológica da Escoriação Psicogênica. 84(5). doi: http://dx.doi.org/10.1590/ S0365-0596008000500016

Ávila, A. L. (2016, agosto) Corpo e mente em questão: em busca da gênese dos sintomas psicossomáticos. Ide (São Paulo), 38(61),51-61, Recuperado em $<$ http:/ / pepsic.bvsalud.org/scielo.phpscript=sci_arttext\&pid=S0101 $-31062016000100005 \& \operatorname{lng}=$ pt\&nrm=iso $>$. Acesso em 21 out. 2019.

Bion, W.R (1970/1991) A Atenção e Interpretação: o acesso científico à intuição em Psicanálise e Grupos. (Rio de Janeiro: Imago. (Trabalho original publicado em 1970).

Campos, E. M. P., Rodrigues, A. L (2005). Mecanismo de formação dos sintomas em psicossomática. Mudanças: Psicologia da saúde, 13(2), 299-312. Doi: https://doi.org/10.15603/2176-1019/mud.v13n2p290-308

Capitão, C. G., Carvalho, E. B. (2006). Psicossomática: duas abordagens de um mesmo problema. Psic: revista da Vetor Editora, 7(2), 21-29. Recuperado em <http://pepsic.bvsalud.org/scielo.php?script=sci_arttext\&pid=S1676$-73142006000200004 \& \operatorname{lng}=$ pt\&tlng=pt.>

Coginotti, I. N. B., REIS, A. H. (2016, dez) Transtorno de escoriação (Skin Picking): revisão de literatura. 12, (2) doi: http://dx.doi.org/10.5935/18085687.20160012 .

Domingues, O. V., Alves, B. A., Colenghi, C. C. S. R., Lima, R. J. F., Pinheiro, N. C. V., Paes, R. C. S \& Silva, F. O. D. (2017, setembro-dezembro) (Skin Picking): um relato de caso. Revista de Medicina e Saúde de Brasilia., 6(3). Recuperado em: <https://portalrevistas.ucb.br/index.php/rmsbr/article/ view/8543>

Feijoo, A. M. L. C. \& Mattar, C. M. (2015). A desconstrução da psicossomática na análise existencial de Heidegger e Boss. Revista Latinoamericana de Psicopatologia Fundamental, 18(4), 651-662. doi: https://dx.doi.org/10.1590/1415$-4714.2015 \mathrm{v} 18 \mathrm{n} 4 \mathrm{p} 651.5$

Freitas, E. P D. (2011) Escoriação psicogênica: aspectos psicológicos e fatores de personalidade. Dissertação (mestrado) - Universidade Estadual Paulista, Faculdade de Ciências, São Paulo.

Garreto, R. K. A. (2015) O desempenho executivo em pacientes que apresentam automutilação. Dissertação (Mestrado em Psiquiatria) - Faculdade de Medicina, Universidade de São Paulo, São Paulo.

Ginsburg, I. H., \& Link, B. H. (1993). Psychosocial consequences of rejection and stigma feelings in psoriasis patients. International Journal of Dermatology, 32 (8), 587-591. doi: <https://doi.org/10.1111/j.1365-4362.1993. tb05031.x>

Giusti, S. J. (2013) Automutilação: características clínicas e comparação com pacientes com transtorno obsessivo-compulsivo. Tese (Doutorado em Psiquiatria) - Faculdade de Medicina, Universidade de São Paulo, São Paulo.

Gomes, R., Ferreira do Nascimento, E., Carvalho de Araujo, F. (2007, março) Por que os homens buscam menos os serviços de saúde do que as mulheres? As explicações de homens com baixa escolaridade e homens com ensino superior. Cad. Saúde Pública., 23(3), 565-574 Recuperado em: $<$ https https://www.scielo.br/scielo.php? script=sci_arttext\&pid=S0102$-311 \mathrm{X} 2007000300015>$.

Grant, J. E., \& Stein, D. J. (2014). Body-focused repetitive behavior disorders in ICD-11. Brazilian Journal of Psychiatry, 36(Suppl. 1), 59-64. doi: https:// dx.doi.org/10.1590/1516-4446-2013-1228

Ibiloğlu, O.; Atli, A. Kaya, M. C., Demir, S., Bulut, M., \& Sir, A. (2016). A Case of Skin Picking Disorder of a Patient with a History of Childhood Abuse. Noro psikiyatri arsivi, 53 (2), 181-1 83.https://doi.org/10.5152/npa.2015.10110

Jorge, H.Z.; Müller, M.C.; Ferreira, V.R.T. \& Cassal, C. (2004). Pacientes portadores de dermatoses: Relações iniciais e auto-agressividade. PSIC - Revista de Psicologia da Vetor Editora, 5 (2), 22-25.

Lovato, L. M. (2011). Skin picking disorder e tricotilomania em adultos portadores de transtorno obsessivo-compulsivo. Dissertação de Mestrado, Faculdade de Medicina, Programa de pós-graduação em psiquiatria, Universidade Federal do Rio Grande do Sul.

Milhorim, T. K., Costa Neto, S. B. (2019) Manifestações corporais do sofrimento psíquico: psicossomática em contexto de pronto-socorro. Revista SBPH. 22(1), 127-153. 
Moher, D., Shamseer, L., Clarke, M., Ghersi, D., Liberati, A., Petticrew, M., Phekelle, P. \& Stewart, L. A. and PRISMA-P Group (2015). PRISMA Group. "Preferred Reporting Items for Systematic Reviens and Meta-Analyses: The PRISMA Statement". Int J Surg, 8, (5) 336-341.

Müller, M. C., Ramos, D. G. (2004). Psicodermatologia: uma interface entre psicologia e dermatologia. Psicologia: ciência e profissão, 24(3), 76-81. Recuperado em 19 de dezembro de 2019, de http://pepsic.bvsalud.org/scielo. php?script $=$ sci_arttext\&pid $=$ S1414-98932004000300010\&lng=pt\&tlng=pt.

Oliveira, E. C. B. (2018) Os aspectos psicopatológicos e fenomenológicos do transtorno de escoriação. Tese (Doutorado em Psiquiatria) - Faculdade de Medicina, Universidade de São Paulo, São Paulo.

Pérez-Elizondo, A. D., Pino-Rojas, G. T. \& Gómez-Espinoza, M. (2013). Excoriaciones psicogénicas: identificación, interpretación y abordaje de una patología de atención compartida. Revista argentina de dermatología, 94(4), Recuperado em: http://www.scielo.org.ar/scielo.php?script=sci_arttext\&pid=S1851-300X2 $013000400004 \& \operatorname{lng}=$ en\&tlng $=$ es.

Ribeiro, I. L. (2011). Espiritualidade nos cuidados de saúde: perspectiva bioética. In: UNIESEP - Núcleo de Investigação em Saúde e Qualidade de Vida. Saúde e qualidade de vida: uma meta a atingir (pp. 320-327). Porto: Escola Superior de Enfermagem do Porto.

Ribeiro, Â., Ribeiro, J. P., \& Von D., (2015). A skin-picking disorder case report: a psychopathological explanation. Jornal Brasileiro de Psiquiatria, 64(2), 177 180. Doi: https://dx.doi.org/10.1590/0047-2085000000075

Saffioti, H. (1987) O poder do macho. São Paulo: SP: Moderna (Coleção polêmica).
Silva, M. A. D. (1994). Quem Ama não Adoece. São Paulo: SP: Círculo do Livro. Silva, J. D. T., Müller, M. C. (2007). Uma integração teórica entre psicossomática, stress e doenças crônicas de pele. Estudos de Psicologia (Campinas), 24(2), 247-256. Doi: https://doi.org/10.1590/S0103-166X2007000200011

Stevanato, D., Prado, R. Martinelli, C. (c2013) A atuação da equipe multiprofissional no diagnóstico e tratamento da escoriação psicogênica. s.n; (25) Recuperado em file:///C:/Users/15-F387WM/Downloads/DANIELE_STEVANATO.pdf

Kim, D. S., Garrison, C. E. \& Thompson, G. (2013). A neta fatal case of pathologicak skin picking. 14, (284-287). Doi: 10.12659/AJCR.889357.

Valente, B. G. (2012) A questão da simbolizacão na psicossomática: estudo com pacientes portadores de transtorno neurovegetativo somatoforme e de transtorno de pânico. Dissertação (Mestrado em Psicologia Clínica) - Instituto de Psicologia, Universidade de São Paulo, São Paulo.

Valerio, C. (2011) Impacto do tratamento do transtorno obsessivo-compulsivo nas comorbidades psiquiátricas no curto e médio prazo. Dissertação (Mestrado em Psiquiatria) - Faculdade de Medicina, Universidade de São Paulo, São Paulo.

Yoshinaga, G. I. \& Galias, I. (2018, dezembro) A pele que somos e a pele que sentimos: Pele - simbolo - consciência. Junguiana, 36, (2), 77-88, recuperado em $<$ http://pepsic.bvsalud.org/scielo.php? script $=$ sci_arttext\&pid $=$ S0103$-08252018000200005 \& \operatorname{lng}=\mathrm{pt \& n} \mathrm{m}=\mathrm{iso}>$

Submetido em: 25-5-2020

Aceito em: 27-1-2021 